\title{
Laser Induced Breakdown Spectroscopy for Feasibility Study of Polymer Aging Test
}

\author{
Kaite Li \\ Department of Electrical Engineering, North China Electric Power University, Baoding 071000, \\ China;
}

5013680247@qq.com

Keywords: Polymer, aging, LIBS

\begin{abstract}
Polymers such as polyethylene (PE), polypropylene (PP), polyvinyl chloride (PVC) are important downstream products of Petrochemical industry, which play an important role in the national economy and the people's livelihood. While, in the process of ageing detection and recycling, there remain something need being improved. Giving effective monitoring to the aging products is one of them, which is one vital step to improving the techniques of recycling polymer. To compensate for what is in want of today's detecting method, this paper proposes to use laser-induced breakdown spectroscopy (LIBS) to have fast, accurately detect of the characters of polymer including PE, PP, LIBS is of great capacity of adapting to environment being detected, covering more detecting elements and almost need no sample prepare.
\end{abstract}

\section{Introduction}

By the comprehensive action of sunlight, oxygen, heat, chemical medium, microorganism and other environmental factors, the chemical composition and structure of polymer would have a series of changes and the performance of polymer would become worse. In order to meet the different needs of production and life, the rapid and accurate detection of the properties of the products is particularly important. However, a large part of the user currently still uses traditional means to detect the density, melt index (or melt flow rate) and other properties of products. The sampling process of traditional means is complex and it has long testing period, which inevitably leads to hysteretic nature, not only serious affects the comprehensive properties of the products, but also greatly reduces the operation safety. Therefore, at present we desiderate a suitable method, especially one on-line monitoring method that can provide feedback signals and achieve system closed circuit control to detect the properties of products [1].

Aiming at these problems, the paper discussed the feasibility of that using laser-induced breakdown spectroscopy which has great capacity of adapting to environment being detected and covering more detecting elements to detect properties of polymers. The laser-induced breakdown spectroscopy technique is a kind of spectroscopy measurement method, using high power laser to irradiate the surface of sample, and then high temperature plasma appears on the surface of the sample because the energy of laser higher than the threshold of the energy needed to produce plasma. Collecting its spectrum signal by spectrometer and then analyzing elemental composition of the sample. Sample pretreatment of laser-induced breakdown spectroscopy is simple and a kind of almost no loss. The technology has become one of the main methods in the field of elemental analysis and has been widely used in the field of environment pollution detection, material element analysis, archaeology, biomedical and so on. It well provides adequate theoretical support for the study in the aspect of on-line detection if gathering laser-induced breakdown spectroscopy of polymers in different using time, analyzing physical chemistry reflected by laser-induced breakdown spectroscopy, making use of the incidence relation between species of elemental, content of elemental and properties of polymers, etc. and building a series of laser-induced breakdown spectroscopy prediction model for properties of polymers [2]. 


\section{Principle and research status of laser-induced breakdown spectroscopy}

The laser-induced breakdown spectroscopy technique is a kind of spectroscopy measurement method, using high power laser to irradiate the surface of sample, and then high temperature plasma appears on the surface of the sample because the energy of laser higher than the threshold of the energy needed to produce plasma. Collecting its spectrum signal by spectrometer and then analyzing elemental composition of the sample. LIBS is a kind of material element analysis technique based on atomic emission spectroscopy, having the advantages of great capacity of adapting to environment being detected, covering more detecting elements, almost need no sample prepare, etc. The technology is widely used in the field of industrial production control based on the particular characteristic of LIBS technique, such as fast, online, simultaneous multicomponent analysis, etc. The LIBS technique is applicable to measure all of the material, because it is an all optical method, and the measurement object does not need to be processed or only need simply pretreatment, so the measurement time is greatly saved, and it also can realize that analyze multielements for measure object at the same time.

In recent years, many research achievements have been obtained by using LIBS technology. The laser-induced breakdown spectroscopy technique has incomparable advantages over other traditional analytical methods in many ways, for example in aspects of fast response, high sensitivity, as well as remote sensing measurement, etc. And these advantages also make the LIBS technology more apply to the detection in complex environment. The main application areas are: environment pollution source monitoring, materials elements analysis, archaeology and sapphire identification science, biomedical, as well as related material elements identification in the area of military [3].

\section{Aging causes of polymers}

\subsection{Effect of light}

It's the solar spectrum in the ultraviolet region cause Initial photochemical process of polymer. Solar spectrum is a continuous spectrum, when passing through the atmosphere was absorbed after the arrival of the earth's surface ultraviolet spectral wavelength in the range of $290-400 \mathrm{~nm}$. This part of the ultraviolet radiation accounts for less than $1 \%$ of the total solar radiation reaching the earth's surface. Although the proportion is not large, its energy has a significant impact on the aging of polymers. Because the energy of the chemical bond breaking in the macromolecular chain is roughly in this range. Polymers containing aldehyde, ketone, carbonyl, peroxide, double bond are more easily absorb the energy of ultraviolet light, causing large molecular chain rupture and aging.)

\subsection{Effect of oxygen and ozone}

The oxidative degradation of polymers is one of the most important factors for the aging of polymers. Ozone has stronger oxidation ability to polymer. Ozone is very unstable and can be easily decomposed atomic oxygen, the oxidation of oxygen than usual much stronger, can direct oxidation of unsaturated hydrocarbons and rubber, the macromolecular chain degradation or crosslinking, decreased the macroscopic physical properties, appearance color become brittle, microcosmic containing oxygen groups.

\subsection{Effect of temperature}

With the increase of temperature, the movement of macromolecular chain is increased, once exceed the dissociation energy, which will cause the decomposition of chain 、 random fault 、 thermal decomposition of side base decomposition, resulting the deterioration rate of polymer is accelerated. The effect of thermal energy can not only make the molecular chain break, but also make the molecular chain cross-linking. Cross link between the polymer chain to generate chemical bonds and increase the molecular weight. However, excessive cross linking makes the materials hard and brittle, and the performance is decreased.

\subsection{Effect of microorganism}

There are many kinds of microorganisms that invade the polymer, mainly the bacteria. Under appropriate conditions of temperature and humidity, the microorganism can grow on the surface of a 
polymer. Any plasticizer and grease compounds containing polymer, especially compounds containing the fatty acid structure is susceptible to mildew. In hot and humid environment, fungal secretions will cause the material to be decomposed into alcohols, organic acids and other substances, these substances for fungal growth provide nourishment, so that mold can be parasitic and reproduce, and the biological degradation intensified.

\section{Experimental device and analysis method}

Experimental device is showed on the picture one, pulse Nd: fundamental frequency $1064 \mathrm{~nm}$ and frequency-doubled 532nm of YAG laser (Continuum, PL8000) as excitation source, laser output pulse width of 8ns, repetition rate of $10 \mathrm{~Hz}$, and laser normal irradiates stainless steel sample through convex lens which is focal length of $25 \mathrm{~cm}$. Position irradiated in the surface is excited and products high temperature plasma. The temperature plasma is allocated into the grating spectrometer (ActonSP2758) through grating spectrometer, and then is handled by spectrometer. Emitting light is transformed from CCD to electrical signal and collecting and handling data is controlled by computer. The resolution of spectrometer is $0.03 \mathrm{~nm}(10 \mu \mathrm{m}$ slit), through the calibration of mercury lamp, and the precision of wavelength is $\pm 0.1 \mathrm{~nm}$. The samples were fixed in a two-dimensional electric displacement platform, and the laser effect at different points in sample surface each time in order to avoid the heterogeneity of the sample caused by the high power laser corrosion.

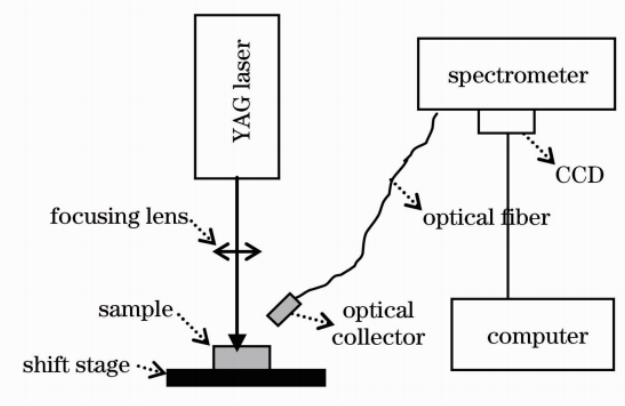

Fig. 1 Experimental Device Diagram

Laser is focused by lens and effect at the surface of stainless steel samples, and sample absorb the energy of laser. Going through the process of heating, melting and gasification, gasified samples absorb the energy of laser unceasingly and form high temperature plasma. The fluorescence of plasma which neutral and charged ion in excited eradiate out forms LIBS after is handled by spectrometer. The type of element can be qualitatively analyzed by the position of wavelength of the characteristic line. According to the National Institute of Standards and Technology (NIST) database atomic spectral line wavelength table and reference spectral lines for attribution.

\section{References}

[1] Rifkin J. The third industrial revolution: how lateral power is transforming energy, the economy and the world [M]. Berlin: Macmillan, 2011: 27-68.

[2] Xu Z, Guan X, Jia X, et al. Performance analysis and comparison on energy storage devices for smart building energy management[J] . IEEE Transactions on Smart Grid, 2012, 3(4): 2136-2147,

[3] Huang Ru, Ye Le, Liao Huailin. Microelectronics technologies in renewable energy internet[J]. China Science: Information Series, 2014, 44(6): 728-742(in Chinese). 